\title{
THE ELECTRONIC STRUCTURE OF THE RMn ${ }_{2} \mathrm{Ge}_{2}$ $(\mathrm{R}=\mathrm{Ca}, \mathrm{Y}, \mathrm{La}, \mathrm{Ba})$ ANTIFERROMAGNETS
}

\author{
J. Toboea ${ }^{a *}$, B. Malaman ${ }^{b}$ And G. Venturini ${ }^{b}$ \\ ${ }^{a}$ Faculty of Physics and Nuclear Techniques, Academy of Mining and Metallurgy \\ Al. Mickiewicza 30, 30-059 Kraków, Poland \\ ${ }^{b}$ Laboratoire de Chimie du Solide Mineral, Université Henri Poincaré-Nancy I, \\ 54506 Vandoeuvre les Nancy Cedex, France
}

The electronic structure of the tetragonal $\mathrm{RMn}_{2} \mathrm{Ge}_{2}(\mathrm{R}=\mathrm{Ca}, \mathrm{Y}, \mathrm{La}, \mathrm{Ba})$ antiferromagnets is presented using the self-consistent Korringa-Kohn-Rostoker method. According to the neutron refinements, two types of collinear antiferromagnetic structure are taken into account: $A F_{1}$ for $\mathrm{YMn}_{2} \mathrm{Ge}_{2}$ and $\mathrm{AF}_{2}$ for the other compounds. The calculated magnetic moments on $\mathrm{Mn}$ : $2.17 \mu_{\mathrm{B}}\left(\mathrm{YMn}_{2} \mathrm{Ge}_{2}\right), 2.84 \mu_{\mathrm{B}}\left(\mathrm{CaMn}_{2} \mathrm{Ge}_{2}\right), 2.95 \mu_{\mathrm{B}}\left(\mathrm{LaMn}_{2} \mathrm{Ge}_{2}\right)$, and $3.47 \mu_{\mathrm{B}}$ $\left(\mathrm{BaMn}_{2} \mathrm{Ge}_{2}\right)$ remain in good agreement with the neutron data (in $\mu_{\mathrm{B}}$ ) 2.20, $2.67,3.05$, and 3.66 , respectively. As seen on antiferromagnetic density of states, all systems are metallic, however $\mathrm{BaMn}_{2} \mathrm{Ge}_{2}$ is found near semimetallic limit. The total energy Korringa-Kohn-Rostoker computations on $\mathrm{CaMn}_{2} \mathrm{Ge}_{2}$, performed in both antiferromagnetic phases, result in preferring of the $\mathrm{AF}_{2}$ structure.

PACS numbers: 71.15.Mb, 71.15.Nc, 75.20.En, 75.20.Hr, 75.50.Ee

\section{Introduction}

The magnetic properties of the well-known ternary $\mathrm{RMn}_{2} \mathrm{Ge}_{2}$ compounds [1] with the tetragonal $\mathrm{ThCr}_{2} \mathrm{Si}_{2}$-type structure have attracted renewed interest in recent years. Detailed neutron diffraction investigations of many $\mathrm{RMn}_{2} \mathrm{Ge}_{2}$ have revised previously detected ferromagnetic (F) structures $(R=L a-N d)[2,3]$ and explored the magnetic behavior of new compounds ( $\mathrm{CaMn}_{2} \mathrm{Ge}_{2}$ and $\mathrm{BaMn}_{2} \mathrm{Ge}_{2}$ [4]). In $\mathrm{LaMn}_{2} \mathrm{Ge}_{2}$, antiferromagnetic ordering $\left(\mathrm{AF}_{2}\right)$ occurs below $T_{\mathrm{N}} \approx 400 \mathrm{~K}$ whereas rather canted magnetic structure than $c$-axis collinear ferromagnetism appears below $T_{\mathrm{C}}$, with the antiferromagnetic component markedly larger $\left(\mu_{\mathrm{AF}}=2.7 \mu_{\mathrm{B}}\right)$ than the ferromagnetic one $\left(\mu_{\mathrm{F}}=1.5 \mu_{\mathrm{B}}\right)$. These results have been later supported by the Mössbauer effect measurements [5]. The three other entitled compounds show purely AF ordering of the Mn magnetic moments. There is a critical distance between $\mathrm{Mn}$ atoms in the $a-b$ plane $\left(d_{\text {in }}=a / \sqrt{2} \approx 2.86 \AA\right)$, where the magnetic properties change drastically (mostly due to the $\mathrm{AF}_{1}-\mathrm{AF}_{2}$ transition).

*corresponding author; e-mail: tobola@ftj.agh.edu.pl, tobola@grxtal.polycnrs-gre.fr 
Thus, in $\mathrm{YMn}_{2} \mathrm{Ge}_{2}\left(d_{\text {in }}=2.82 \AA\right) \mu_{\mathrm{Mn}}$ are arranged parallel in the $\mathrm{Mn}$ plane and coupled $A F$ between the planes $\left(A F_{1}\right.$-type) [6], while in $\mathrm{CaMn}_{2} \mathrm{Ge}_{2}$ ( $d_{\text {in }}=$ $2.94 \AA)$ and $\mathrm{BaMn}_{2} \mathrm{Ge}_{2}\left(d_{\mathrm{in}}=3.14 \AA\right)$ the neighboring $\mu_{\mathrm{Mn}}$ are aligned antiparallel both in and between the manganese planes $\left(\mathrm{AF}_{2}\right.$-type) [4]. Ishida et al. [7] have calculated the electronic structure of the "ferromagnetic" $\mathrm{LaMn}_{2} \mathrm{Ge}_{2}$ by the Korringa-Kohn-Rostoker (KKR) method. However, the theoretical values of $\mu_{\text {tot }}$ and $\mu_{\mathrm{Mn}}$ have been found rather in disagreement with experimental data available at this time. Furthermore, Kulatov et al. [8] have discussed magnetic ordering of $\mathrm{RMn}_{2} \mathrm{Si}_{2}$ and magnetic transition near $d_{\text {in }} \approx 2.86 \AA$, using the non-polarized linear muffin-tin orbital (LMTO) results.

The purpose of this paper is to show the electronic structure and magnetism of $\mathrm{RMn}_{2} \mathrm{Ge}_{2}$ using experimentally detected magnetic ordering of $\mu_{\mathrm{Mn}}$.

\section{Computational details}

The spin-polarized calculations on the tetragonal body-centered (bct) $\mathrm{RMn}_{2} \mathrm{Ge}_{2}$ compounds are performed by the spin and charge self-consistent KKR method $[9,10]$ within the local spin density framework (the exchange-correlation potential given by the von Barth-Hedin formula [11]). In the $\mathrm{RMn}_{2} \mathrm{Ge}_{2}$ structure $(I 4 / \mathrm{mmm}) \mathrm{R}, \mathrm{Mn}$, and Ge atoms occupy $2(\mathrm{a})[0,0,0], 4(\mathrm{~d})[0,1 / 2,1 / 4]$ and 4(e) $[0,0, z]$ Wyckoff sites, respectively. The crystallographic data measured at $T=2 \mathrm{~K}[2-4]$ and muffin-tin radii $r_{\mathrm{R}}: \mathrm{r}_{\mathrm{Mn}}: r_{\mathrm{Ge}} \approx 1.5: 1: 1$ (giving $\sum V_{\mathrm{mt}}^{i} / V_{\mathrm{Ws}} \approx$ $67 \%)$ are used in the KKR computations. For the final potentials $\left(\Delta E_{\text {tot }} \approx 1 \mathrm{mRy}\right)$ the total densities of states (DOS), site-decomposed DOS and $l$-decomposed DOS (with $l_{\max }=2$ for $\mathrm{R}=\mathrm{Ca}, \mathrm{Y}, \mathrm{Ba}$ and $l_{\max }=3$ for $\mathrm{R}=\mathrm{La}$ ) are computed. The relativistic effects are incorporated in calculations of core levels in $\mathrm{BaMn}_{2} \mathrm{Ge}_{2}$ and $\mathrm{LaMn}_{2} \mathrm{Ge}_{2}$. Note that in $\mathrm{YMn}_{2} \mathrm{Ge}_{2}\left(\mathrm{AF}_{1}\right)$, no more bct symmetry is maintained, then the KKR calculations are performed within the simple tetragonal structure. Integration in the $k$-space is performed using 192 small tetrahedrals and $135 k$-points in the irreducible part of the Brillouin zone.

\section{Results and discussion}

The main KKR results for $\mathrm{RMn}_{2} \mathrm{Ge}_{2}$, calculated both in $\mathrm{F}$ and AF states, are summarised in Table. The theoretical values of $\mu_{\mathrm{Mn}}$ remain in close agreement with the neutron diffraction data, recently measured at $T=2 \mathrm{~K}$ [2-4]. The magnetisation $\left(4.22 \mu_{\mathrm{B}}\right)$ as well as the $\mathrm{Mn}$ magnetic moments $\left(2.29 \mu_{\mathrm{B}}\right)$ found in the ferromagnetic $\mathrm{LaMn}_{2} \mathrm{Ge}_{2}$ are also close to the earlier theoretical values $\left(3.9 \mu_{\mathrm{B}}\right.$ and $2.1 \mu_{\mathrm{B}}$ ) [7]. From comparison of the $\mathrm{F}$ and AF results one notes that $\mu_{\mathrm{Mn}}$ in the $\mathrm{AF}_{2}$-type compounds are rather larger than these computed in the $\mathrm{F}$ state (particularly in $\mathrm{LaMn}_{2} \mathrm{Ge}_{2}$ ), while they are almost the same in $\mathrm{YMn}_{2} \mathrm{Ge}_{2}\left(\mathrm{AF}_{1}\right.$-type ordering).

If plotting the theoretical $\mu_{\mathrm{Mn}}$ versus $d_{\text {in }}$ in the $\mathrm{RMn}_{2} \mathrm{Ge}_{2}$ antiferromagnets (Fig. 1) we observe that the Mn magnetic moment increases more or less proportionally to $d_{\text {in }}$, which well corresponds to the phenomenological relations established among $d_{\text {in }}, \mu_{\mathrm{Mn}}$ and magnetic ordering type [12]. Nevertheless, one should take care using this simple model (Fig. 1), since in real samples when lattice constants increase, $z_{\mathrm{Ge}}$ also changes. A number of the KKR computations on 
the $\mathrm{RMn}_{2} \mathrm{X}_{2}$ systems, carried out with the use of different $a, c$ and $z_{\mathrm{X}}$ parameters, lead to conclusion that the $z_{\mathrm{X}}$ value (like $d_{\text {in }}$ ) has a strong influence on a magnitude of $\mu_{\mathrm{Mn}}$ in $\mathrm{RMn}_{2} \mathrm{X}_{2}$. Moreover, both $z_{\mathrm{X}}$ and $d_{\text {in }}$ seem crucial in appearing of magnetic properties in $\mathrm{RT}_{2} \mathrm{X}_{2}$ (with $\mathrm{T} \neq \mathrm{Mn}$ ) [13].

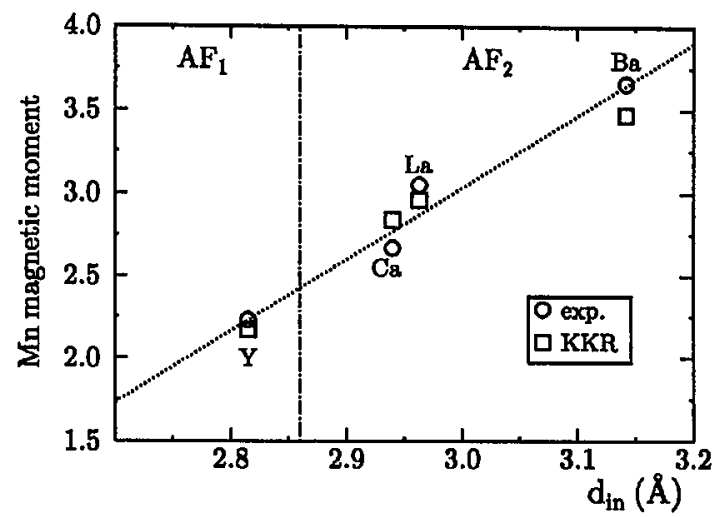

Fig. 1. The $\mathrm{Mn}$ magnetic moment (in $\mu_{\mathrm{B}}$ ) versus the $\mathrm{Mn}-\mathrm{Mn}$ distance $\left(d_{\mathrm{in}}\right)$ in $\mathrm{RMn}_{2} \mathrm{Ge}_{2}$.

\section{TABLE}

The KKR results in $\mathrm{RMn}_{2} \mathrm{Ge}_{2}$ (magnetic moments in $\mu_{\mathrm{B}}, \mathrm{DOS}$ in states/Ry/spin).

\begin{tabular}{c|c|c|c|c}
\hline \hline & $\mathrm{R}=\mathrm{Y}$ & $\mathrm{R}=\mathrm{Ca}$ & $\mathrm{R}=\mathrm{La}$ & $\mathrm{R}=\mathrm{Ba}$ \\
\hline \multicolumn{5}{c}{$\mathrm{F}$ state } \\
\hline$\mu_{\text {tot }}$ & 4.02 & 4.99 & 4.22 & 6.78 \\
$\mu_{\mathrm{Mn}}$ & 2.15 & 2.59 & 2.29 & 3.33 \\
$n_{\uparrow}\left(E_{\mathrm{F}}\right)$ & 18.79 & 18.69 & 20.16 & 27.55 \\
$n_{\downarrow}\left(E_{\mathrm{F}}\right)$ & 32.80 & 51.42 & 65.52 & 41.14 \\
\hline \multicolumn{5}{c}{$\mathrm{AF}$ state } \\
\hline $\mathrm{KKR}$ & 2.17 & 2.84 & 2.95 & 3.48 \\
exp. & 2.20 & 2.67 & 3.05 & 3.66 \\
$n_{\uparrow \downarrow}\left(E_{\mathrm{F}}\right)$ & $47.51^{*}$ & 10.96 & 48.99 & 3.06 \\
\hline "per two formula units
\end{tabular}

The DOS of $\mathrm{YMn}_{2} \mathrm{Ge}_{2}\left(\mathrm{AF}_{1}\right)$ (Fig. 2) consists of two broad peaks coming mostly from the $\mathrm{Mn}$ sites. Unlikely, the DOS of $\mathrm{CaMn}_{2} \mathrm{Ge}_{2}, \mathrm{LaMn}_{2} \mathrm{Ge}_{2}$, and $\mathrm{BaMn}_{2} \mathrm{Ge}_{2}\left(\mathrm{AF}_{2}\right)$ presents complex structure with few narrow $d$-like peaks arising also on $\mathrm{Mn}$ atoms (note that the highest $d$-like peaks are localized well below $E_{\mathrm{F}}$ in contrast to $d$-DOS in $\mathrm{YMn}_{2} \mathrm{Ge}_{2}$ ). The conduction band in $\mathrm{RMn}_{2} \mathrm{Ge}_{2}$ is formed mostly by $d$-states on $\mathrm{Mn}$ and $\mathrm{R}$ with some admixture of $p$-states on Ge. 

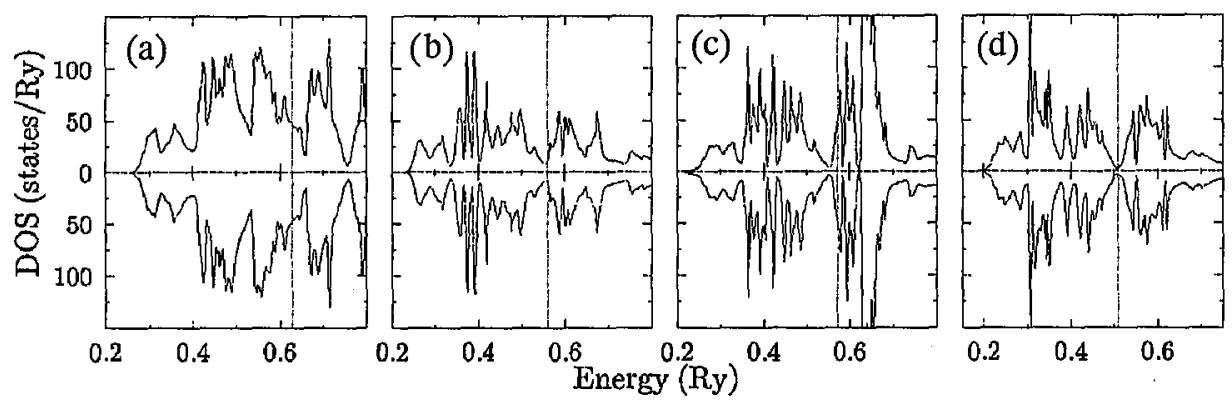

Fig. 2. The total DOS in the $\mathrm{RMn}_{2} \mathrm{Ge}_{2}$ antiferromagnets: (a) $\mathrm{YMn}_{2} \mathrm{Ge}_{2}$, (b) $\mathrm{CaMn}_{2} \mathrm{Ge}_{2}$, (c) $\mathrm{LaMn}_{2} \mathrm{Ge}_{2}$, (d) $\mathrm{BaMn}_{2} \mathrm{Ge}_{2}$.

The s-states (not plotted in Fig. 2) form two separate bands and are located 0.13-0.15 Ry below the conduction band bottom. When passing from $R=Y$ to $\mathrm{R}=\mathrm{Ba}$ (increasing $d_{\mathrm{in}}$ ), less overlapping of $d$-like wave functions occurs, giving rise to narrow bands. Consequently, $\mathrm{Mn}$ atoms keeps successively larger magnetic moment reaching the $3.48 \mu_{\mathrm{B}}$ value ( $3.66 \mu_{\mathrm{B}}$ from experiment) in $\mathrm{BaMn}_{2} \mathrm{Ge}_{2}$. Interestingly, from the KKR calculations this compound is found near the semimetallic limit (Fig. 2). The $E(k)$ computations (not shown) result in two strongly dispersive bands crossing the Fermi level, while an energy gap occurs along most of $k$-vector directions. As $\mathrm{CaMn}_{2} \mathrm{Ge}_{2}$ and $\mathrm{BaMn}_{2} \mathrm{Ge}_{2}$ have the same number of valence electrons, the low DOS at $E_{\mathrm{F}}$ is also detected in $\mathrm{CaMn}_{2} \mathrm{Ge}_{2}$. In $\mathrm{LaMn}_{2} \mathrm{Ge}_{2} E_{\mathrm{F}}$ is shifted into the DOS peak due to one more electron. To investigate a reason for the changing of the AF-type structure, near $d_{\text {in }} \approx 2.86 \AA$ the total energy KKR computations are done on $\mathrm{CaMn}_{2} \mathrm{Ge}_{2}$ applying $\mathrm{AF}_{1}$ and $\mathrm{AF}_{2}$. Indeed, $E_{\text {tot }}$ has a slightly lower value when using the $\mathrm{AF}_{2}$ structure. But the similar comparison in $\mathrm{YMn}_{2} \mathrm{Ge}_{2}$ does not give reliable preference for $\mathrm{AF}_{1}$. Then, more detailed analysis $E_{\mathrm{tot}}=f\left(a, c, z_{\mathrm{Ge}}\right)$ should be undertaken to clarify this point.

\section{Acknowledgments}

This work has been realized during the fellowship granted to J.T. from the Henri Poincaré University in Nancy. The computations have been partly performed in the Supercomputing Center-Cyfronet in Kraków.

\section{References}

[1] A. Szytuła, J. Leciejewicz, in: Handbook on Physics and Chemistry of Rare Earth, Vol. 12, Eds. K.A. Gschneidner Jr., L. Eyring, Elsevier, Amsterdam 1989, p. 133.

[2] G. Venturini, R. Welter, B. Malaman, J. Alloys Comp. 210, 213 (1994).

[3] G. Venturini, R. Welter, E. Ressouche, B. Malaman, J. Alloys Comp. 223, 101 (1995).

[4] B. Malaman, G. Venturini, R. Welter, E. Ressouche, J. Alloys Comp. 210, 209 (1994).

[5] I. Nowik, Y. Levi, I. Felner, E.R. Bauminger, J. Magn. Magn. Mater. 140-144, 913 (1995). 
[6] A. Szytuła, I. Szott, Solid State Commun. 40, 199 (1981).

[7] S. Ishida, S. Asano, J. Ishida, J. Phys. Soc. Japan 55, 936 (1986).

[8] E. Kulatov, V. Veselago, L. Vinokurova, Acta Phys. Pol. A 77, 709 (1990).

[9] A. Bansil, S. Kaprzyk, J. Toboła, in: Applications of Mutiple Scattering Theory in Material Science, Vol. 252, Eds. W.H. Butler, P.H. Dederichs, A. Gonis, R.L. Weaver, MRS, Pittsburg 1992, p. 505.

[10] S. Kaprzyk, Acta Phys. Pol. A 91, 135 (1997).

[11] U. von Barth, L. Hedin, J. Phys. C 5, 1629 (1972).

[12] G. Venturini, B. Malaman, E. Ressouche, J. Alloys Comp. 241, 135 (1996).

[13] $\mathrm{YCr}_{2} \mathrm{Si}_{2}$ has been predicted to be magnetic from the $\mathrm{KKR}$ method, which is now confirmed by the neutron diffraction measurements (I. Ijjaali, G. Venturini, B. Malaman, J. Alloys Comp. 279, 102 (1998)). 